Cite this: RSC Adv., 2014, 4, 26354

Received 13th March 2014 Accepted 4th June 2014

DOI: $10.1039 / c 4 r a 04547 e$

www.rsc.org/advances

\section{Semi-crystalline block copolymer bicontinuous nanospheres for thermoresponsive controlled release $\uparrow$}

\author{
Simon J. Holder, ${ }^{* a}$ Glen Woodward, ${ }^{a}$ Beulah McKenzie ${ }^{\text {ab }}$ \\ and Nico A. J. M. Sommerdijk ${ }^{\text {b }}$
}

We demonstrate the controlled release of pyrene, as a model hydrophobic molecule, from self-assembled bicontinuous nanospheres formed from an amphiphilic block copolymer. The bicontinuous polymer nanospheres act as efficient nanocarriers and the incorporation of hydrophobic poly(alkyl methacrylate) blocks introduces a temperature responsive component to the hydrophobic core.

Block copolymer self-assembled aggregates have long been studied as nanocarriers for the controlled delivery of encapsulated chemicals, with particular focus on the controlled delivery of pharmaceuticals in biomedical applications. To date most research has focussed on the use of spherical micelles and vesicles (polymersomes) with some studies utilising cylindrical micelles. ${ }^{1,2}$ We have recently demonstrated the existence of selfassembled bicontinuous polymer nanospheres (BPNs) from amphiphilic block copolymers with low weight fractions of hydrophilic blocks $(<25 \%) \cdot{ }^{3-5}$ Whilst micelles and vesicles have primarily been studied for the encapsulation and release of lipophilic and hydrophilic compounds respectively, BPNs offer the potential for dual release formulations (e.g. a lipophilic and a hydrophilic pharmaceutical) stemming from the coexistence of the hydrophilic and hydrophobic interior regions. Herein we present the results from our studies into successfully encapsulating a hydrophobic compound (pyrene) into such nanospheres, and the subsequent controlled release of pyrene as a function of temperature.

Thermoresponsive polymers, macromolecules that undergo a physical property change in response to a change in

\footnotetext{
${ }^{a}$ Functional Materials Group, University of Kent, Canterbury, CT2 8EN, UK. E-mail: S.J.Holder@kent.ac.uk; Tel: +441227823542

${ }^{b}$ Laboratory of Materials and Interface Chemistry and Soft Matter Cryo-TEM Research Unit, Eindhoven University of Technology, P.O. Box 513, 5600 MB Eindhoven, The Netherlands

$\dagger$ Electronic supplementary information (ESI) available: Experimental methods and techniques; Py in water fluorescence spectra and calibration curve; diffusion modelling fitting plots for PEO-PODMA nanospheres, micelles and pure Py; TEM of PEO-PODMA micelles. See DOI: 10.1039/c4ra04547e
}

temperature, have been proposed for a number of potential applications. One of the most common being for controlled release from discrete self-assembled aggregates such as micelles and vesicles in biomedical and pharmaceutical uses., ${ }^{1,2}$ Most of these thermoresponsive systems have been based upon poly( $N$-isopropylacrylamide) and poly(oligoethylene glycol $)^{6}$ and other hydrophilic polymers. ${ }^{7}$ Upon heating above a given temperature (the lower critical solution temperature) such materials become insoluble. A different approach to utilising thermoresponsive polymers has been described using poly(trimethylene carbonate) wherein a melting transition for a hydrophobic polymer results in a profound change in permeability. ${ }^{8,9}$ Unfortunately polymers for which crystallinity is a property of the main chain have limitations, particularly with respect to manipulation of the melting temperature. A promising approach to the use of semi-crystalline polymers for temperature responsive applications is the incorporation of the crystalline segment as a component of the side-chain; i.e. removed from the backbone. ${ }^{10}$ By changing the alkyl chain length the melting temperature can be modulated. ${ }^{11}$ The poly(alkyl (meth)acrylates) are ideal candidates for this approach. ${ }^{12}$ In this study we demonstrate the potential of this alternative whereby we use poly(octadecyl methacrylate) as semi-crystalline polymer component in a block copolymer bicontinuous nanosphere and demonstrate the release of pyrene across a range of temperatures from 10 to $40{ }^{\circ} \mathrm{C}$.

We prepared poly(ethylene oxide)-block-poly(octadecyl methacrylate) (PEO- $b$-PODMA) bicontinuous nanospheres in aqueous solution by the slow addition of deionised water to a THF solution of a $\mathrm{PEO}_{45}-b$-PODMA 20 copolymer $\left(M_{\mathrm{n}}=10900\right.$, $M_{\mathrm{w}} / M_{\mathrm{n}}=1.26$, by GPC) followed by dialysis against water at $10{ }^{\circ} \mathrm{C}$ (ESI $\dagger$ ). ${ }^{5}$ The resulting BPNs displayed a bicontinuous internal structure of interpenetrating PODMA and PEO-water domains with average pore diameters of $20 \mathrm{~nm}$ as characterised by cryo-TEM and cryo-ET. The resulting size distribution was monomodal with a $z$-average diameter of $180 \pm 3 \mathrm{~nm}$, a numberaverage diameter of $127 \pm 8 \mathrm{~nm}$ and polydispersity index of 0.27 by DLS and $235 \pm 16 \mathrm{~nm}$ by TEM (Fig. 1a, c and ESI $\dagger$ ). BPNs 


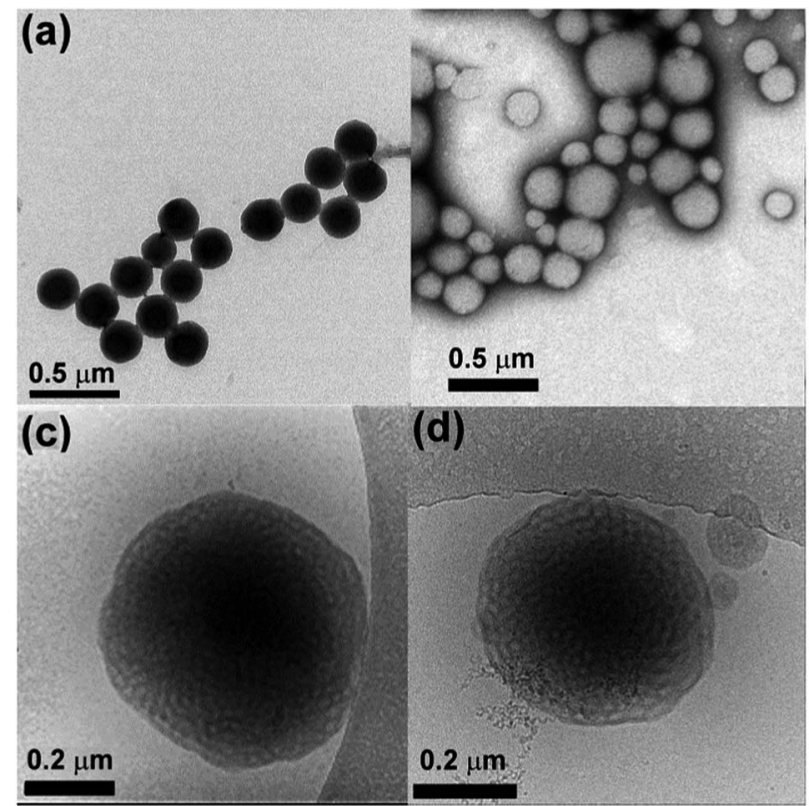

Fig. 1 TEM micrographs of PEO- $b$-PODMA micelles (a) with no pyrene (no staining), (b) with pyrene encapsulated (negative staining). Cryo-TEM micrographs of bicontinuous nanospheres (c) without pyrene and (d) with pyrene, illustrating internal morphology.

resemble cubosomes generated by molecular amphiphiles which are usually stabilised by PEO based additives. ${ }^{\mathbf{1 3}}$ Such cubosomes have been proposed and studied for a number of controlled delivery applications. ${ }^{\mathbf{1 4 , 1 5}}$ Whilst our bicontinuous polymeric nanospheres have lower degrees of order than those of their molecular analogues they are easily prepared, their components (PEO biocompatible corona and alkyl chain interiors) are intrinsic to the structure and open to ready modification, and crucially they are kinetically stable. By following identical preparation conditions but using a THF solution of pyrene (Py) in place of pure THF we were able to form bicontinuous nanospheres with encapsulated Py with a monomodal distribution with a $z$-average diameter of $268 \pm 23 \mathrm{~nm}$, a number-average diameter of $141 \pm 37 \mathrm{~nm}$, a polydispersity index of 0.27 by DLS (number average) and an average diameter of $222 \pm 86 \mathrm{~nm}$ by TEM (Fig. 1b, $\mathrm{d}$ and ESI $\dagger$ ). Py was chosen as a model for the controlled release of hydrophobic compounds owing to its hydrophobic character with very low water solubility (typically in the range $2 \times 10^{-7}$ to $10 \times 10^{-7} \mathrm{~mol} \mathrm{dm}^{-3}$ ). ${ }^{16,17}$ Since a dialysis (at $10^{\circ} \mathrm{C}$ ) was involved in the preparation, it was necessary to demonstrate that a significant amount of Py was still present and retained in the nanospheres. This was demonstrated by analysis of the fluorescent spectra of the Py encapsulated PEO-PODMA solution with that of a Py solution prepared in the absence of the copolymer (Fig. 2). Three aspects of the emission spectra demonstrated encapsulation: (i) a significant difference in emission intensities for otherwise identical quantities of Py, indicative of solubilised Py; (ii) the comparison of the spectra gave $I_{3}: I_{1}$ ratios $\left(I_{1}\right.$ is the $0-0$ vibronic band at $372 \mathrm{~nm}$ and $I_{3}$ is the $0-737$ band at $383 \mathrm{~nm}$ ) of 1.03 for the encapsulated Py and 0.67 for the Py in water, indicative of Py

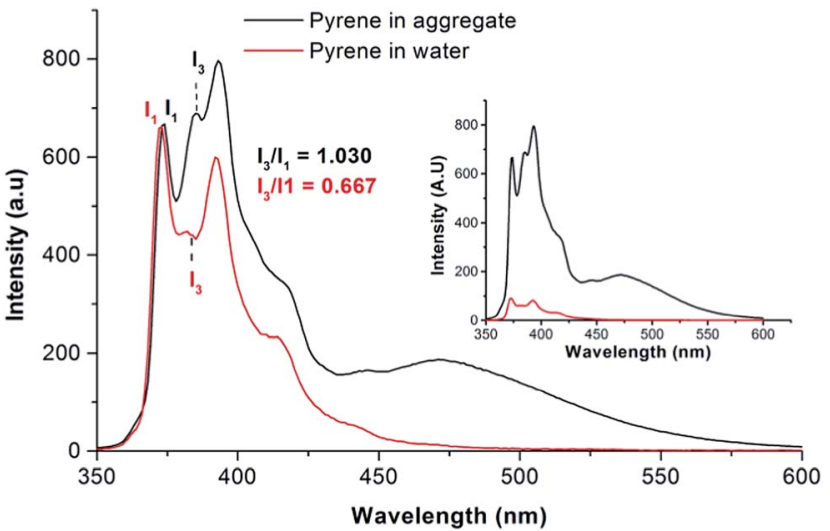

Fig. 2 Normalised fluorescence emission spectra of pyrene in water (red line) and encapsulated in bicontinuous micelle (black line). The inset shows the spectra prior to normalisation.

in a relatively weakly polar environment for the micellar dispersion, and a highly polar environment in water; ${ }^{5,18,19}$ (iii) a strong excimer emission $\left(\lambda_{\max }=471 \mathrm{~nm}\right)$ for the Py in the micellar dispersion; indicative of localised high concentrations of Py.

We previously reported that upon heating from $5{ }^{\circ} \mathrm{C}$ to $45{ }^{\circ} \mathrm{C}$ a phase transition at $20-25{ }^{\circ} \mathrm{C}$ was observed for the bicontinuous components of the PODMA regions of the block copolymer. ${ }^{5}$ To study the release of Py into the aqueous environment upon heating, samples of the Py-PEO-PODMA dispersion were isolated from pure water $\left(3 \mathrm{dm}^{3}\right)$ by a dialysis membrane and kept at a set temperature $\left(10,20,25,30\right.$ and $\left.40{ }^{\circ} \mathrm{C}\right)$. The relative quantity of Py released into the larger aqueous environment was determined by recording fluorescent spectra of samples at given times over a 6 to 7 hour period (Fig. 3a). A calibration curve of Py in water was used to determine Py concentration variance with time (see ESI $\dagger$ ). To confirm that the release of the Py was occurring from the nanospheres the BPN solutions were also analysed by fluorescence spectroscopy after dialysis. In all cases a clear decrease in emission intensity was observed corresponding to loss of pyrene from the nanospheres (Fig. 3b). The release efficiencies were estimated from integration of the curves relative to the undialysed pyrene containing BPN sample (see ESI $\dagger$ ). As can be seen (Fig. 3b) a relative maximum in release efficiency ( $72 \%$ release of encapsulated pyrene) was observed at $40{ }^{\circ} \mathrm{C}$. These results also allowed an estimate of the loading capacity and gave a value of $43 \mu \mathrm{g}$ pyrene per mg of BPN (circa 4\% loading). A full study of release efficiency involving infinite sink conditions is needed and is currently underway.

The release profiles for $10{ }^{\circ} \mathrm{C}, 20{ }^{\circ} \mathrm{C}$ and $25{ }^{\circ} \mathrm{C}$ are very similar, but for $30-40{ }^{\circ} \mathrm{C}$ there is an increasing rate of release of Py (Fig. 3a). The plot of fractional release against time from 10 to $60 \%$ release (see $\mathrm{ESI} \dagger$ ) is linear indicating zero order kinetics and Case-II transport properties (a non-Fickian diffusion release mechanism predominates). Fractional release times were based on extrapolation of the curves in Fig. 3a to constant values resulting from saturation of the water solution by Py which varies with temperature (see ESI $\dagger$ ). ${ }^{16}$ The change in release rates (taken from the slope of the linear fits, ESI $\dagger$ ) show 


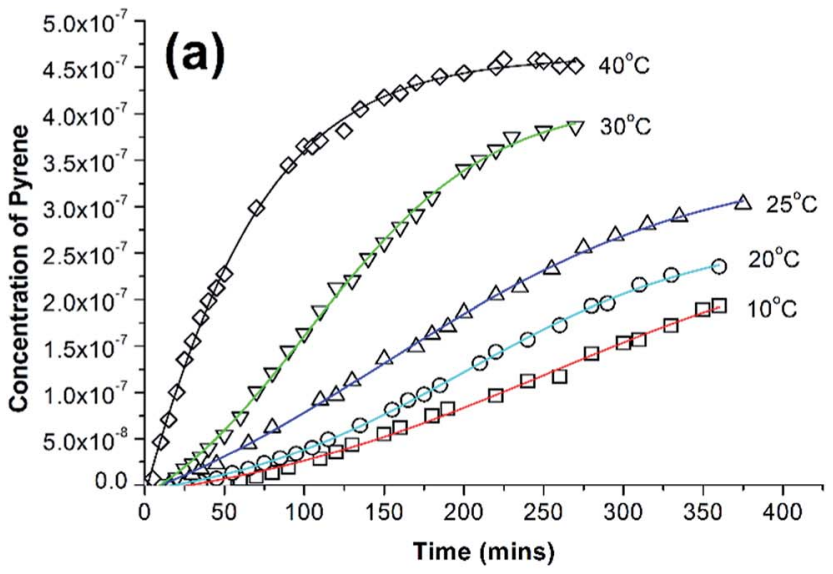

(b)

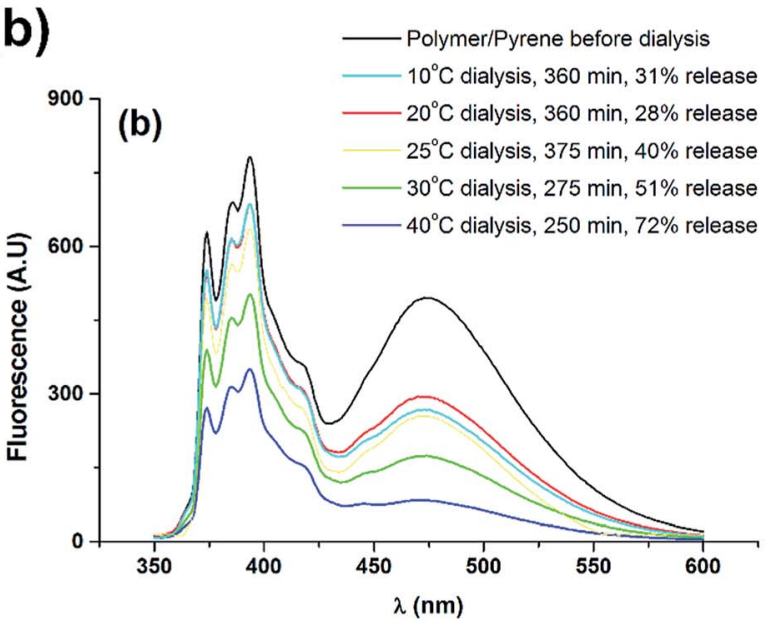

Fig. 3 Release of pyrene from bicontinuous nanospheres formed from $\mathrm{PEO}_{45}-b$-PODMA 20 , (a) release profiles at various temperatures and (b) photoluminescent spectra of Py nanosphere dispersions before and after dialysis at stated temperatures.

the dramatic change from below $25^{\circ} \mathrm{C}$ to above $25^{\circ} \mathrm{C}$ (Fig. 4a), with fractional release rate constants of $0.0030 \mathrm{~s}^{-1}$ at $25^{\circ} \mathrm{C}$ and $0.0051 \mathrm{~s}^{-1}$ at $30^{\circ} \mathrm{C}$ and $0.01003 \mathrm{~s}^{-1}$ at $40^{\circ} \mathrm{C}$. This demonstrates the effect of the melting of the hydrophobic PODMA block on Py release and the potential of poly(alkyl methacrylates) in thermoresponsive controlled delivery applications.

As has become increasingly apparent over the past few years, dynamic equilibrium dialysis conditions are not always suitable for reliably determining the release kinetics of lipophilic compounds from solid nanoparticles and micelles. ${ }^{20-24}$ To a large part the apparent release kinetics in such set-ups are a result of the partition coefficient between particle and lipophilic compound within the dialysis chamber, any binding to the dialysis membrane (potentially complicated by nanoparticle effects on the membrane itself) and diffusion rates across the dialysis membrane any and all of which can be the limiting factor in the rate of appearance of the lipophile in the receiving chamber. This can complicate data interpretation and can lead to incorrect conclusions about the release kinetics. ${ }^{25}$ As a control experiment, an identical procedure was followed whereby the release of Py from a dispersion prepared without the PEO-PODMA present was monitored. Since Py is only
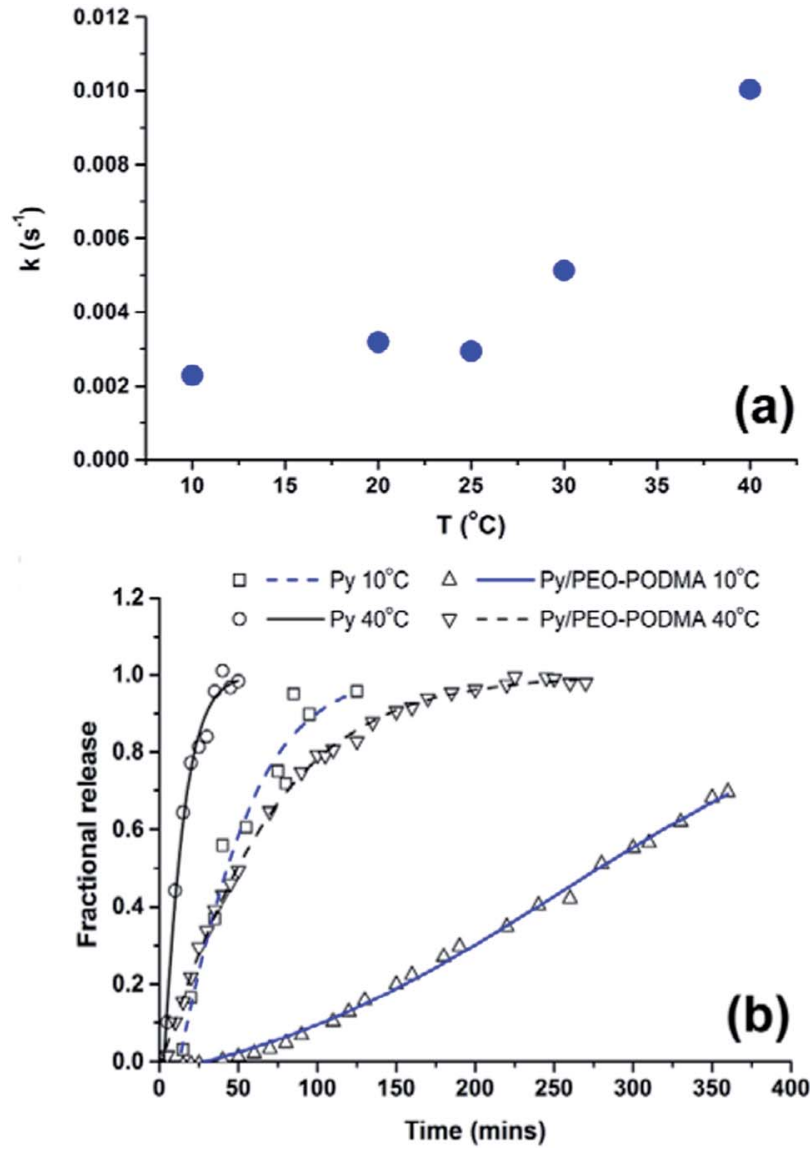

Fig. 4 (a) Time taken for indicated percentage release of pyrene from bicontinuous nanospheres at given temperatures. (b) Comparison of fractional release profiles of 'free' pyrene (see text) and pyrene encapsulated in bicontinuous nanospheres.

sparingly water soluble the bulk of the Py remained undissolved. Transfer of the solid Py-water mixture to dialysis tubing and monitoring of the release profiles showed that release of Py into surrounding water was significantly faster than that of the encapsulated Py at $40{ }^{\circ} \mathrm{C}$ and at $10{ }^{\circ} \mathrm{C}$ (Fig. 4b). However the difference in release rates for 'free' and encapsulated pyrene and change in rates with temperature for the encapsulated pyrene indicate a controlled release mechanism.

Despite the reservations currently held about the full validity of dynamic equilibrium dialysis in studying release mechanisms, a number of drug dissolution models were fitted to the fractional release profiles namely the Higuchi, zero order, Weibull and Korsmeyer-Peppas (power law) models. ${ }^{26-31}$ of these the Korsmeyer-Peppas model most closely matched the release profiles (between 10 and $60 \%$ release) (see ESI $\dagger$ ). This model takes the form

$$
\frac{M_{t}}{M_{\infty}}=a t^{n}
$$

where $M_{t} / M_{\infty}$ is the fraction released at time $t, a$ is a constant incorporating structural and geometrical characteristics of the drug dosage form and $n$ is the release exponent which is indicative of the release mechanism. A release exponent $(n)$ of 
0.43 for a spherical geometry indicates a release mechanism following Fickian diffusion, $0.43<n<0.85$ indicates an anomalous diffusion mechanism (where encapsulant release is primarily determined by polymer relaxation rates) and $n>0.85$ Case-II transport where release rate is determined predominantly by polymer relaxation processes in non-swellable and non-degradable systems. By comparing the $n$ across the temperatures (see ESI $\dagger$ ) it was observed that from $10-30{ }^{\circ} \mathrm{C}$ the exponent $n$ took values $>1.0$ indicative of Super Case-II transport occurring where polymer relaxation properties were the determining factor for the release rates over Fickian diffusion determined by Py concentration gradients. ${ }^{32}$ This is in line with transport mechanisms observed for non-swellable and nondegradable synthetic polymers. ${ }^{32-34}$ The exact location of the Py in the BPNs is uncertain. The fluorescence data (Fig. 2) demonstrates that the majority of the Py is not simply adsorbed onto the surface of the spheres (a substantially lower $I_{3} / I_{1}$ ratio would be expected as a consequence of the polarity of the immediate environment). ${ }^{18}$ Thus there are four possible internal regions in the BPNs that can be considered as sites for the Py; (i) the $\mathrm{PEO}+\mathrm{H}_{2} \mathrm{O}$ region, (ii) the PEO-PODMA interfacial region, (iii) the PODMA amorphous polar backbone region, (iv) the crystalline octadecyl region) (Scheme 1). The $\mathrm{PEO}-\mathrm{H}_{2} \mathrm{O}$ region can be discounted since the observed $I_{3} / I_{1}$ ratio is much higher than would be expected and the octadecyl crystalline region can be discounted due to the lower $I_{3} / I_{1}$ ratio than expected (Fig. 2) and it is known that chromophores are excluded from the crystalline regions in polymers. ${ }^{19}$ Thus the Py can be postulated to reside predominantly in the PEO-PODMA interfacial region and/or the PODMA backbone region. The observed $I_{3} / I_{1}$ ratio precludes neither of these possibilities. That the rate of release increases significantly above $T_{\mathrm{m}}$ of PODMA is in line with the previously observed dramatic increases in gas permeability above $T_{\mathrm{m}}$. The increase in permeability is directly attributable to a decrease in backbone stiffness in wholly amorphous state compared to the semi-crystalline state. Thus for the BPNs we suggest that above $T_{\mathrm{m}}$ the relaxation rates of the PODMA chains increase and diffusion through the polymer is greatly enhanced.

In summary we have demonstrated that self-assembled bicontinuous nanospheres formed from amphiphilic block copolymers can be used to encapsulate and therefore transport hydrophobic compounds in water. They have been demonstrated to display controlled release and furthermore the release

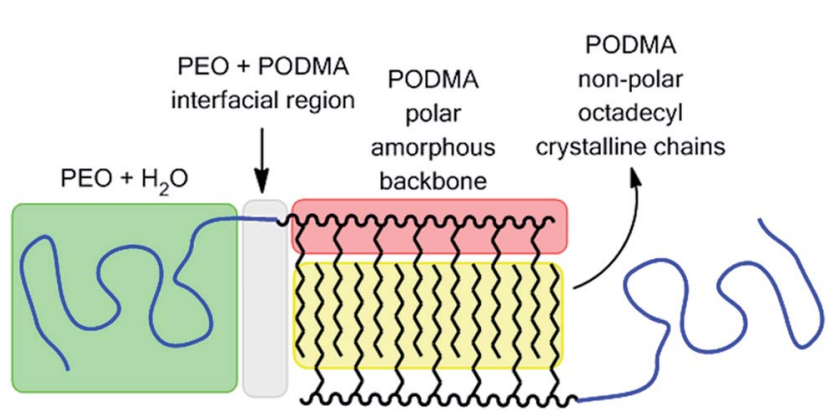

Scheme 1 Illustration of potential regions for pyrene inclusion in BPN. rate can be significantly increased through an increase in temperature.

Finally we note that the melting temperatures of the poly(alkyl(meth)acrylates) are readily altered through the use of different lengths of alkyl chains as substituents and our current work is investigating raising the transition temperatures to greater than $30^{\circ} \mathrm{C}$.

\section{Notes and references}

1 A. Rösler, G. W. M. Vandermeulen and H.-A. Klok, Adv. Drug Delivery Rev., 2012, 64, 270-279.

2 U. Kedar, P. Phutane, S. Shidhaye and V. Kadam, Nanomedicine: Nanotechnology, Biology and Medicine, 2010, 6, 714-729.

3 B. E. McKenzie, J. F. de Visser, H. Friedrich, M. J. M. Wirix, P. H. H. Bomans, G. de With, S. J. Holder and N. A. J. M. Sommerdijk, Macromolecules, 2013, 46, 98459848.

4 B. E. McKenzie, S. J. Holder and N. A. J. M. Sommerdijk, Curr. Opin. Colloid Interface Sci., 2012, 17, 343-349.

5 B. E. McKenzie, F. Nudelman, P. H. Bomans, S. J. Holder and N. A. Sommerdijk, J. Am. Chem. Soc., 2010, 132, 10256-10259.

6 H. Wei, S.-X. Cheng, X.-Z. Zhang and R.-X. Zhuo, Prog. Polym. Sci., 2009, 34, 893-910.

7 S. Hocine and M.-H. Li, Soft Matter, 2013, 9, 5839-5861.

8 C. Sanson, O. Diou, J. Thevenot, E. Ibarboure, A. Soum, A. Brûlet, S. Miraux, E. Thiaudière, S. Tan and A. Brisson, ACS Nano, 2011, 5, 1122-1140.

9 C. Sanson, J. F. Le Meins, C. Schatz, A. Soum and S. Lecommandoux, Soft Matter, 2010, 6, 1722-1730.

10 G. Street, D. Illsley and S. Holder, J. Polym. Sci., Part A: Polym. Chem., 2005, 43, 1129-1143.

11 K. O'Leary and D. R. Paul, Polymer, 2004, 45, 6575-6585.

12 E. Hempel, H. Huth and M. Beiner, Thermochim. Acta, 2003, 403, 105-114.

13 P. T. Spicer, Curr. Opin. Colloid Interface Sci., 2005, 10, 274279.

14 C. Y. Guo, J. Wang, F. L. Cao, R. J. Lee and G. X. Zhai, Drug Discovery Today, 2010, 15, 1032-1040.

15 S. B. Rizwan, B. J. Boyd, T. Rades and S. Hook, Expert Opin. Drug Delivery, 2010, 7, 1133-1144.

16 F. P. Schwarz, J. Chem. Eng. Data, 1977, 22, 273-277.

17 T. A. Andersson, K. M. Hartonen and M.-L. Riekkola, J. Chem. Eng. Data, 2005, 50, 1177-1183.

18 D. C. Dong and M. A. Winnik, Can. J. Chem., 1984, 62, 25602565.

19 S. Abraham, T. D. Atvars and R. G. Weiss, J. Phys. Chem. B, 2010, 114, 12221-12233.

20 B. J. Boyd, Int. J. Pharm., 2003, 260, 239-247.

21 C. Washington, Int. J. Pharm., 1989, 56, 71-74.

22 Y. Zambito, E. Pedreschi and G. Di Colo, Int. J. Pharm., 2012, 434, 28-34.

23 H. Bunjes, J. Pharm. Pharmacol., 2010, 62, 1637-1645.

24 C. Washington, Int. J. Pharm., 1990, 58, 1-12.

25 S. Modi and B. D. Anderson, Mol. Pharm., 2013, 10, 30763089. 
26 P. Costa and J. M. Sousa Lobo, Eur. J. Pharm. Sci., 2001, 13, 123-133.

27 T. Higuchi, J. Pharm. Sci., 1963, 52, 1145-1149.

28 C. G. Varelas, D. G. Dixon and C. A. Steiner, J. Controlled Release, 1995, 34, 185-192.

29 F. Langenbucher, J. Pharm. Pharmacol., 1972, 24, 979-981.

30 W. Weibull, J. Appl. Mech., 1951, 18, 293-297.
31 R. W. Korsmeyer, R. Gurny, E. Doelker, P. Buri and N. A. Peppas, Int. J. Pharm., 1983, 15, 25-35.

32 Y. Fu and W. J. Kao, Expert Opin. Drug Delivery, 2010, 7, 429444.

33 D. De Kee, Q. Liu and J. Hinestroza, Can. J. Chem. Eng., 2005, 83, 913-929.

34 S. C. George and S. Thomas, Prog. Polym. Sci., 2001, 26, 9851017. 